\title{
Experimental study of an inclined jet-in-cross-flow interacting with a vortex generator
}

\author{
K.B.M.Q. Zaman ${ }^{1}$, D. L. Rigby ${ }^{2}$ and J. D. Heidmann ${ }^{3}$ \\ NASA Glenn Research Center \\ Cleveland, OH 44135
}

\begin{abstract}
An experiment is conducted on the effectiveness of a vortex generator (VG) in preventing lift-off of a jetin-cross-flow (JICF), with film-cooling application in mind. The jet issues into the boundary layer at an angle of $20^{\circ}$ to the free-stream. The effect of a triangular ramp-shaped VG is studied while varying its geometry and location. Detailed flow-field properties are documented for a specific case in which the height of the VG and the diameter of the orifice are comparable to the approach boundary layer thickness. This combination of VG and JICF produce a streamwise vortex pair with vorticity magnitude three times larger (and of opposite sense) than that found in the JICF alone. Such a VG appears to be most effective in keeping the jet attached to the wall. While most of the data are taken at a jet-to-freestream momentum flux ratio $(J)$ of 2 , limited surveys are done for varying $J$. The VG is found to have a significant effect even at the highest $J(=11)$ covered in the experiment. Effect of parametric variation is studied mostly from surveys ten diameters downstream from the orifice. When the VG height is halved there is a lift-off of the jet. On the other hand, when the height is doubled, the jet core is dissipated due to larger turbulence intensities. Varying the location of the VG, over a distance of three diameters from the orifice, is found to have little impact. Rounding off the edges of the VG with increasing radius of curvature progressively diminishes the effect. However, a small radius of curvature may be quite tolerable in practice.
\end{abstract}

\section{Introduction}

A vortex generator geometry for boundary layer flow control, sometimes referred to as 'micro-ramp', has been addressed in recent studies [1-3]. With certain orientation, the VG produces a pair of streamwise vortices whose sense is such that there is a downwash between the two vortices and the free-stream fluid is ingested and drawn towards the wall. This sense is opposite to that of the 'bound vortex pair' occurring in the JICF that tends to cause the jet to lift away from the wall. Thus, placing the VG in front of an inclined JICF, typical of film-cooling application, has the promise of keeping the jet attached to the wall via vorticity cancellation. The basic concept is not new and has been tried before with other methods of streamwise vortex generation [e.g., Ref. 4]. The present VG geometry for film-cooling purposes was explored in the CFD study of [1] that showed promising results. This prompted the current experiments to further explore the potential of the method. Detailed flow-field data are acquired for an isolated JICF issuing at an angle of $20^{\circ}$ to the free-stream. The location and height of the VG as well as the momentum flux ratio are varied. Furthermore, since sharp-edged VG may not be easy to implement in practice, the effect of rounding off the edges of the VG is also examined. Hot-wire anemometry is used to measure mean

\footnotetext{
${ }^{1}$ Inlet \& Nozzle Branch, Aeropropulsion Division, AIAA Associate Fellow.

${ }^{2}$ ASRC Aerospace Inc, NASA GRC.

${ }^{3}$ Turbomachinery and Heat Transfer Branch, Aeropropulsion Division, AIAA member.
} 
velocity, turbulence and streamwise vorticity characteristics while varying different parameters. These results are documented and discussed in this paper.

\section{Experimental Facility and Procedure}

The measurements are carried out in a low speed, open-loop wind tunnel having a 20 in. high $\mathrm{x}$ 30 in. wide test section [5]. The experimental arrangement and the VG models are shown in Fig.1. The jet orifice is a round hole of diameter $d=0.75$ in., inclined at an angle of $20^{\circ}$. The orifice plate, flush mounted on the floor of the test section, has a thickness of $1 \mathrm{in}$. The supply for the jet is shop air that goes through a flow conditioning screen into a settling chamber before issuing into the test section through the orifice [5]. The jet velocity $\left(U_{j}\right)$ is monitored by a pressure transducer in the line. The pressure transducer is calibrated by measuring the velocity (using a Pitot probe) at the exit of a 0.75 in. diameter orifice that is cut perpendicular to the surface of another identical plate. As it will be seen in the following, the velocity distribution at the exit of the inclined orifice in highly non-uniform, thus, $U_{j}$ represents an average velocity. Unless stated otherwise, the data pertain to a nominal free-stream velocity $\left(U_{\infty}\right)$ of $29 \mathrm{ft} / \mathrm{s}$ and jet velocity $\left(U_{j}\right)$ of $41 \mathrm{ft} / \mathrm{s}$, yielding a momentum flux ratio $J\left(=\left(U_{j} / U_{\infty}\right)^{2}\right)$ of 2 .

The vortex generator and its location relative to the orifice are shown schematically in Fig. 2. Detailed surveys are conducted for the VG model denoted 'H0 (R0)' in Fig. 1(c). It has dimensions L, S and $\mathrm{H}$ of $1.435,1.18$ and 0.561 inches, respectively. The half-angle at the apex $\left(22.5^{\circ}\right)$ is somewhat smaller than that used in [1] but closer to that used in [2]. Various other VG models used in the study are shown in Fig. 1(c). Their essential dimensions are listed in table 1. Note that when varying the radius of curvature of the edges of the VG (models R0 thru R4) the maximum height and the frontal area blockage were kept constant. The limiting model in this series is ' $\mathrm{R} 4$ ' that is a hemisphere with a small cylindrical base. Most of the data taken are for the VG placed with its apex upstream ('film-cooling' configuration), as shown in Figs. 1 and 2. Limited data with a reversed configuration (apex downstream or the "mixing enhancement' configuration) are also taken and discussed briefly at the end of the paper.

$\begin{array}{cc}\text { VG } & H(\text { in }) \\ \text { H0 } & 0.561 \\ \text { H1 } & 1.125 \\ \text { H2 } & 0.281 \\ (L=1.435 \text { in, } S=1.18 \text { in, } \rho=0.005 \text { in }) \\ \text { VG } & \rho \text { (in) } \\ \text { R0 } & 0.005 \\ \text { R1 } & 0.032 \\ \text { R2 } & 0.141 \\ \text { R3 } & 0.250 \\ \text { R4 } & 0.340 \\ \left(H=0.561 \text { in, Area blockage }=0.33 \mathrm{in}^{2}\right)\end{array}$

Table 1 Dimensions of VG models (Fig. 1c); $\rho$ is the radius of curvature at the edges of the VG.

The profile of the turbulent approach boundary layer is shown in Fig. 3. The boundary layer thickness $(\delta \approx 0.6 d)$ is approximately comparable to the height of the R series models $(H=0.75 d)$. The 
Reynolds number $(R e)$ based on freestream velocity and the orifice diameter is 11,400 . Both $R e$ and $\delta / d$ are approximately the same as used in the CFD study of [1].

A single hot-wire is used for conducting preliminary checkouts as well as over-the-orifice measurements (Fig. 1a). Two X-wires, one in $u-v$ and another in $u$ - $w$ configuration (Fig. 1b), are used for detailed flow-field surveys downstream of the VG. The surveys are conducted on $y$-z planes at various $x / d$ locations. Detailed surveys are done with the model 'H0(R0)' placed with its tip at $x / d=1$. The surveys are done at $x / d=3.1,5,10$ and 15 - the first location being at the 'foot', i.e., at the left edge of the VG (Fig. 2). With parametric variations, the surveys are conducted only at $x / d=10$, for diagnosing the relative effect. Data for most cases are taken for three flow conditions: (a) only JICF without the VG, (b) only VG without the JICF and (c) combination of VG and JICF. Note that while data for all components of mean velocity and turbulent stresses have been acquired, for brevity only data for streamwise mean velocity, turbulence intensity and vorticity are presented. Furthermore, only examples of flow-field survey contours are shown while overall trends are summarized by line graphs.

\section{Results}

The mean velocity and turbulence intensity distribution over the orifice are shown in Fig. 4. The two plots in the upper row represent data for only the jet flow with the tunnel flow turned off. The plots in the lower row show corresponding data with the addition of the tunnel flow. A slight asymmetry remained in the jet despite efforts to remedy it by tracking its source. As it will be seen in the following discussions the effect of the VG dominates the flow and the asymmetry in the jet may be considered inconsequential.

Figure 5 compares the evolution of the mean velocity for the three flow conditions noted in the previous section. These data are for the model 'H0(R0)' with its tip at $x / d=1$ and for $J=2$. The surveys are for four axial locations as indicated. Note that the walls of the test section are $20 d$ away on either side and thus any wall-effect must be negligible even at the last station. The 'dome' of high-speed fluid seen for the JICF alone (first column) is pulled down towards the wall by the VG (third column), especially at the downstream locations. Thus, in film-cooling applications the VG should be beneficial since the coolant flow (JICF) is brought closer to the wall. This occurs in spite of the fact that turbulence intensities, shown in Fig. 6, are increased considerably for the VG-plus-JICF case (see also profiles of u'-peak later). The mean velocity and turbulence intensity distributions at farther downstream locations are seen to be quite similar between the VG-only (second column) and the VG-plus-JICF (third column) cases. This suggests that the effect of the VG might be 'too strong' and a smaller VG might have been sufficient. This issue is addressed further in the following.

The streamwise vorticity distributions, corresponding to the data of Figs. 5 and 6, are compared in Fig. 7. Of note is the fact that the magnitudes for the VG or VG-plus-JICF cases are much larger compared to those for the JICF-only case; note also the sign change. With stronger magnitudes for the VG, the vortices spread much more in the lateral direction. With the addition of the JICF the spread reduces somewhat relative to the VG-only case.

The overall characteristics seen in Figs. 5-7 are captured in the profiles and trajectories shown in Figs. 8-11. The variation of peak streamwise vorticity is shown in Fig. 8. The initial magnitude for the VG-plus-JICF case is somewhat smaller relative to the VG-only case - perhaps, as expected since there is some vorticity cancellation for the combined case. The vortices from the VG are clearly much stronger, 3 times or more in magnitude, relative to the vortices of the JICF case. If the vortices from the VG were of the same magnitude as those of the JICF (but opposite sign), would the cancellation be maximum? This is not yet clear and is discussed further in the following. 
The trajectories of the streamwise vortex cores are shown in Fig. 9. The lateral spreading is more with the stronger vortices for the VG cases relative to the JICF-only case. Fig. 10 shows the drop-off of the peak turbulence intensity with downstream distance. When the JICF is combined with the VG the initial peak levels are much larger. These levels become about the same as that for the VG-only case farther downstream. While the VG clearly pulls the JICF towards the wall, the high initial turbulence may have an adverse effect from a film-cooling perspective. The JICF (i.e., coolant flow) core might dissipate faster due to turbulent diffusion. In fact, an effect like this can be seen in Fig. 5. While the core is brought closer to the wall (in (c)), the spatial extent of the core (dark red region, say, at $x / d=10$ ) is smaller than that of the JICF-only case (in (a)). The trajectories of the peak mean velocity at $\mathrm{z}=0$, approximately read off from Figs. 5(a) and (c), are shown in Fig. 11. It shows the 'jet penetration' and provides a clearer idea about how much the JICF (coolant) core has been drawn towards the wall by the VG. It is needless to say that while the influence of the VG may appear too good from these simplistic graphs the flowfield in its entirety (Fig. 5) must be considered for the overall effect.

The effect of placing the VG ('H0(R0)') at different distances from the orifice is shown in Fig. 12. These and the rest of the surveys shown in the following are done at $x / d=10$. Mean velocity, turbulence intensity and streamwise vorticity are shown in the three columns, as indicated in the caption. The top row represents data taken with the apex of the VG placed right at the end of the orifice; the bottom row represents the farthest location of the VG relative to the orifice. It is apparent that varying the location of the VG did not produce a large change for any of the properties at the measurement station. The overall trends for the vorticity field are captured in Figs. 13 and 14. As the VG is moved downstream, $\omega_{\mathrm{x}}$ cores are drawn somewhat closer together (Fig. 13) and the magnitudes increase (Fig. 14); perhaps, this is expected since the measurement station becomes closer to the VG. However, these changes are much smaller relative to those produced by variation of other parameters.

The effect of varying the momentum flux ratio $(J)$ over a range of 1-11 is explored. Unfortunately, the control for the jet flow was coarse and additional data on the lower end of the range, more typical of film-cooling applications, could not be taken. The parameter $J$ was varied by varying $U_{\infty}$ while keeping $U_{j}$ a constant. First, data for the JICF-only case are shown in Fig. 15 for three values of $J$, as examples. With increasing $J$, the jet lift-off is more, turbulence intensity is increased and the cores of the streamwise vortices become larger. Corresponding data for VG-plus-JICF case are shown in Fig. 16. It is remarkable that even at the highest $J$, the jet core is brought significantly closer to the wall by the action of the VG. The trends are summarized in Fig. 17 showing the magnitude and location of the peak of $U$ at $z=0$, as a function of $J$. It can be seen that the magnitude changes little, however, the location is brought closer to the wall at all values of $J$.

All data presented so far pertained to the model 'H0(R0)'. Data taken for two other VG heights (cases $\mathrm{H} 1$ and H2, table 1), are shown next in Figs. 18 and 19. For clear comparison, the data for the intermediate height (case H0, Figs. 5-7) are repeated in these figures. For VG-only case (Fig. 18), it can be seen that the height has a profound effect. The extent of the velocity defect, turbulence intensity as well as the magnitude of the streamwise vortices, have increased with increasing $H$. Corresponding data for the VG-plus-JICF case (Fig. 19) exhibit similar trends. With reference to the earlier discussion of Fig. 8 , we note that the smallest height case has produced streamwise vortices that are comparable in magnitude but opposite in sense relative to the JICF-only case (compare plot at top of Fig. 18c with data for $x / d=10$ in Fig. 7a). Yet, with the addition of the JICF the vortices from the VG have changed very little (compare plots at top of Figs. 18c and 19c). Furthermore, with these weaker vortices there is less effect on the flow-field and the jet core (top of Fig. 19a) is seen to have lifted off. On the other hand, for the VG with the largest height (at the bottom of Fig. 19a), the jet core has practically diffused due to effect from large turbulence. The increasing turbulence with increasing VG height can be seen in the middle columns 
(Figs. 18b and 19b). Thus, for the range covered, the intermediate height $(H=0.75 d)$ appears to be most effective in pulling the jet towards the wall while not dissipating it adversely.

Finally, the effect of rounding off the edges of the VG is shown in Figs. 20-22. Contour plots for the VG-only case are shown in Fig. 20. These data are for the R1, R2 and R4 models with the relative radii of curvature being indicated. It is noteworthy that even with the limiting hemispherical case (R4; $\rho / \rho_{4}=1$ ) a streamwise vortex pair of same (downwash) sense is produced albeit with smaller magnitude. Corresponding data for the VG plus JICF case are shown in Fig. 21. It is apparent that with the jet on, the flow patterns have changed for the R4 model. The jet flow has overwhelmed the effect of the VG and the basic feature of the JICF has taken over (see, data for JICF-only case in Figs. 5-7). Thus, a vortex pair with small magnitude but upwash sense has prevailed in Fig. 21(c). Streamwise vorticity data have been reported rarely in the literature for comparable flows. Reference [6] reports detailed velocity measurements behind a bump placed in a turbulent boundary layer and some similarity of the turbulence field between the present data (Fig. 20) and theirs may be observed. The 'downwash' vortex pair (at the bottom of Fig. 20c) suggests that even a hemispherical model should be successful in pulling the jet down towards the wall to some extent. This corroborates the observation in [7] that a 'deposition' in front of the cooling orifice produces a beneficial effect.

The streamwise vorticity magnitudes for the R-series models are analyzed in Fig. 22. Peak $\omega_{\mathrm{x}}$ values are shown as a function of the radius of curvature of the edges. (The average of two absolute peaks from a pair is taken for these data - with the downwash sense being assigned a positive and upwash sense a negative sign). Data for cases with a reversed placement of the VG are included in this figure. The VGonly case is represented by the circular symbols with the solid lines. Open points are for the film-cooling configuration while the solid points are for the reversed configuration. As expected, the reversed configuration produces vortex pairs of opposite sense (negative ordinate). It can be seen that the absolute magnitudes of peak $\omega_{\mathrm{x}}$ are significantly larger in the film-cooling configuration. It is as if the flow favors the downwash sense, producing a pair of that sense even in the limiting hemispherical case.

The dashed curves with the diamond symbols in Fig. 22 represent corresponding data for the VGplus-JICF cases. Obviously, with the addition of the JICF there is vorticity cancellation in the filmcooling configuration but augmentation with the reversed configuration, resulting in a downward shift of the entire loop. For the limiting hemispherical model (data on far right) the magnitude has dropped more than that for other radii. However, the magnitude is quite small bordering on measurement uncertainty and too much emphasis must not be given to the exact trends. Nonetheless, it is amply clear that the rounding off of the edges has a significant effect. With increasing roundedness there is decreasing amplitude of $\omega_{\mathrm{x}}$ and, therefore, less effectiveness of the VG. A small amount of rounding (e.g. with model R1, table 1), however, may be quite tolerable with minimal impact on the effectiveness of the VG.

\section{Conclusions}

Experimental results on the interaction of a vortex generator with an inclined jet-in-cross-flow are presented in this paper. The VG produces a streamwise vortex pair that has a sense opposite to that of the bound vortex pair in the JICF. Thus, placing the VG in front of the JICF has a vorticity cancellation effect with a resultant retardation in the tendency for the jet to lift off from the wall. A VG model with dimensions comparable to the orifice diameter and the approach boundary layer thickness is found to produce streamwise vorticity approximately three times larger in magnitude than that found with the JICF alone. This configuration appears to be most effective in keeping the flow attached to the wall. Measuring the flow field at ten diameters from the orifice the following observations are made. When the height of the VG is halved there is a lift-off of the jet. On the other hand, when the height is doubled, the jet core is found to be dissipated due to larger turbulence intensities. Varying the location of the VG, over three 
diameters from the orifice, has little impact on the flow field at the measurement station. It is found that even at the highest value of $J(=11)$ covered in the experiment the VG is quite effective in pulling the jet towards the wall. Finally, it is found that the edges of the VG need to be sharp in order to be effective. With increasing radius of curvature of the edges the vortices weaken and thus the VG become less effective. However, a small radius of curvature may be quite tolerable.

\section{Acknowledgement}

This work is supported by the Subsonic Fixed Wing Project of the NASA Fundamental Aeronautics Program.

\section{References:}

[1] Rigby, D.L. and Heidmann, J.D., "Improved film cooling effectiveness by placing a vortex generator downstream of each hole", Paper 2008-GT-51361, Proc. ASME Gas Turbine Conference, June 9-13, 2008, Berlin, Germany.

[2] Hirt, S.M. and Anderson, B.H., "Experimental investigation of the application of microramp flow control to an oblique shock interaction", AIAA Paper 2009-919, 47 ${ }^{\text {th }}$ Aerospace Sciences Meeting, January 5-8, Orlando, FL.

[3] Ford, C.W.P. and Babinsky, H., "Micro-ramp control of oblique shock wave / boundary layer interactions", AIAA Paper 2007-4115, 37 ${ }^{\text {th }}$ Fluid Dynamics Conf., June 25-28, Miami, FL.

[4] Zaman, K.B.M.Q., 1998, "Reduction of jet penetration in a cross-flow by using tabs", $34^{\text {th }}$ Joint Propulsion Conference, July 13-15, Cleveland, OH, AIAA Paper 98-327.

[5] Milanovic, I.M. and Zaman, K.B.M.Q., 2005, "Synthetic Jets in Crossflow", AIAA J., 43(5), pp. 929-940.

[6] Byun, G. and Simpson, R.L., 2005, "Structure of three-dimensional separated flow on an axisymmtric bump", 43 ${ }^{\text {rd }}$ Aerospace Sciences Meeting, Reno, NV, January 10-13, AIAA Paper 20050113.

[7] Sundaram, N. and Thole, K.A., 2006, "Effects of surface deposition, hole blockage, and TBC spallation on vane endwall film-cooling", ASME Turbo Expo, Barcelona, Spain, May 8-11, Paper GT-90379. 


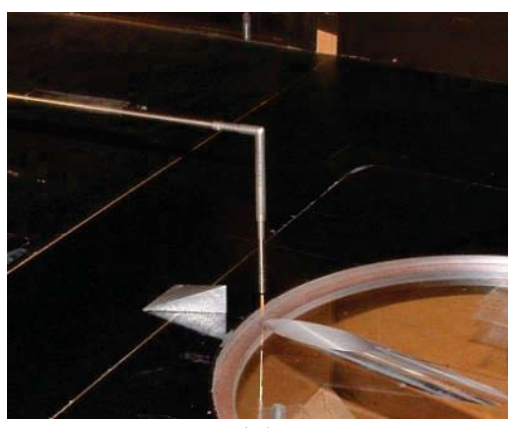

(a)

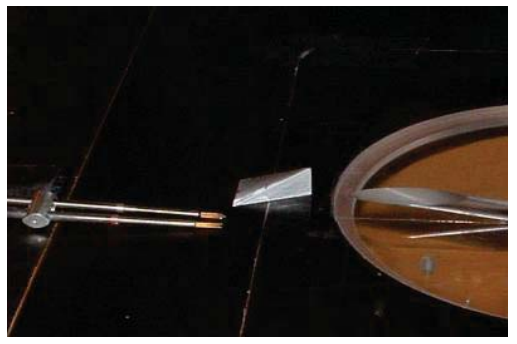

(b)

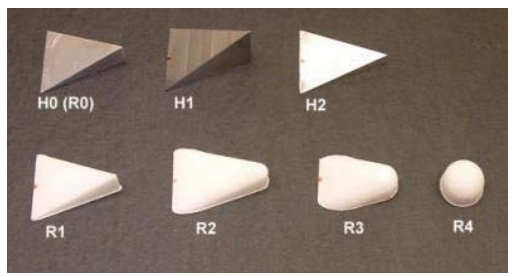

(c)

Fig. 1 Experimental setup in the Wind tunnel. (a) Orifice and vortex generator (VG) with single hotwire, (b) two X-wire set-up, (c) VG models tested.
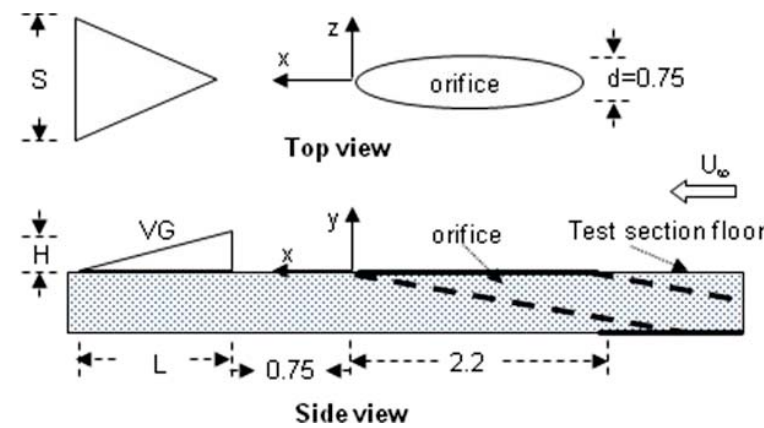

Fig. 2 Schematic of VG and orifice with coordinate system. Dimensions are in inches.

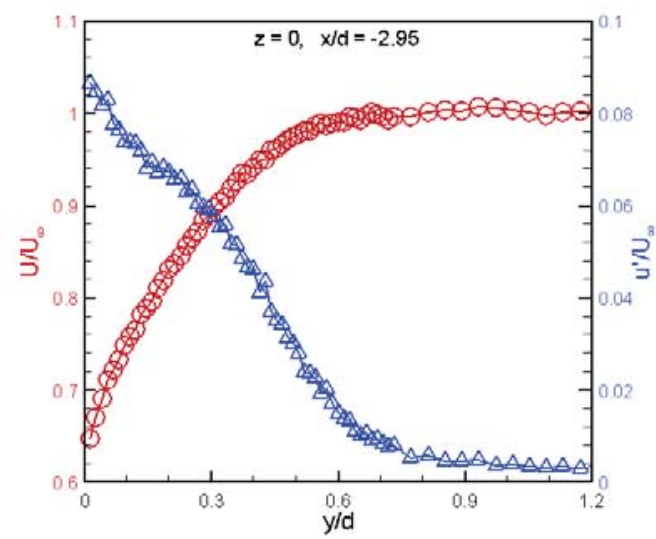

Fig. 3 Approach boundary layer profiles; $U_{\infty}=29 \mathrm{ft} / \mathrm{s}$.
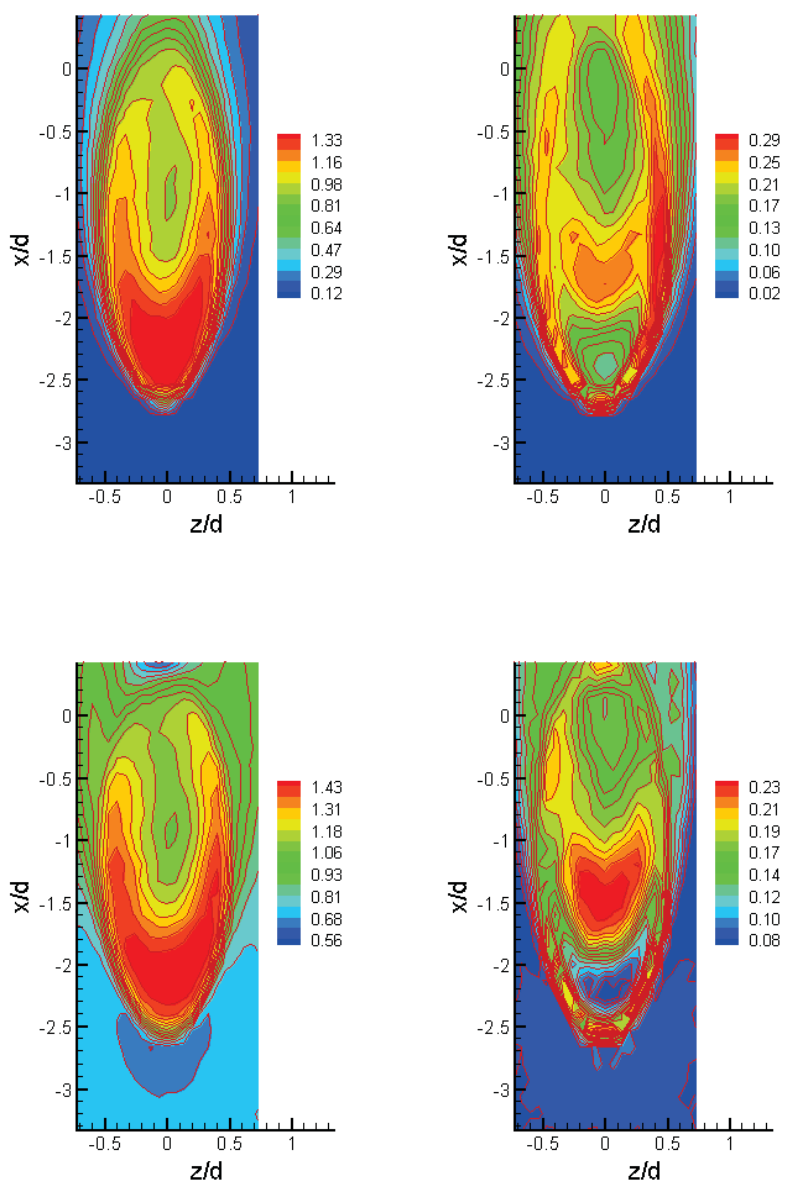

Fig 4 Mean velocity (left) and r.m.s turbulent velocity (right) at orifice exit; $y / d=0.04$. Velocities are normalized by a reference velocity of $29 \mathrm{ft} / \mathrm{s}$. Top row: $U_{\infty}=0, U_{j}=41 \mathrm{ft} / \mathrm{s}$; bottom row: $U_{\infty}=29 \mathrm{ft} / \mathrm{s}, U_{j}=41 \mathrm{ft} / \mathrm{s}$. 

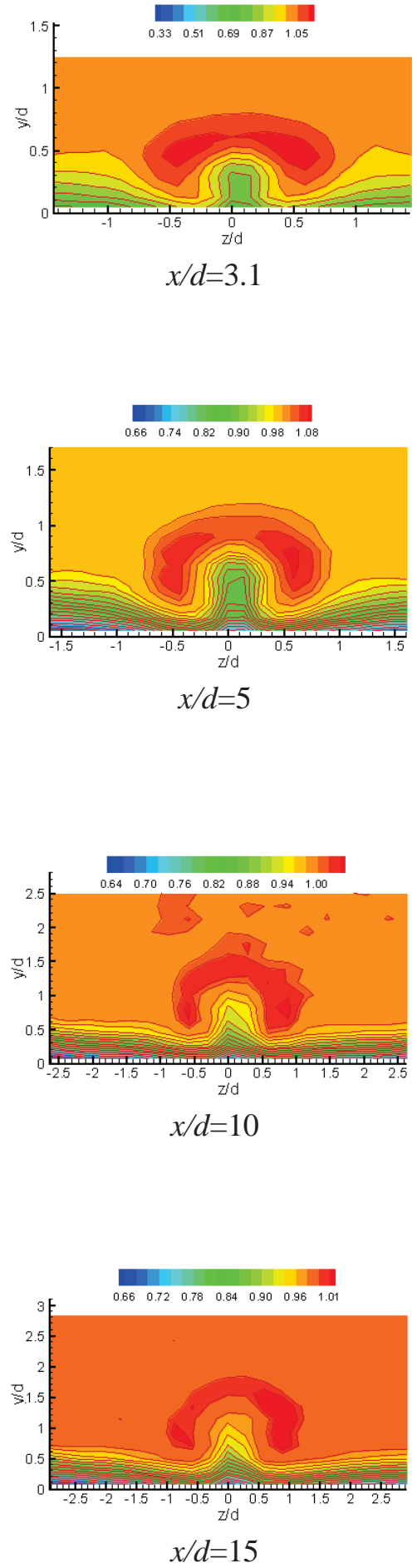

(a) JICF only
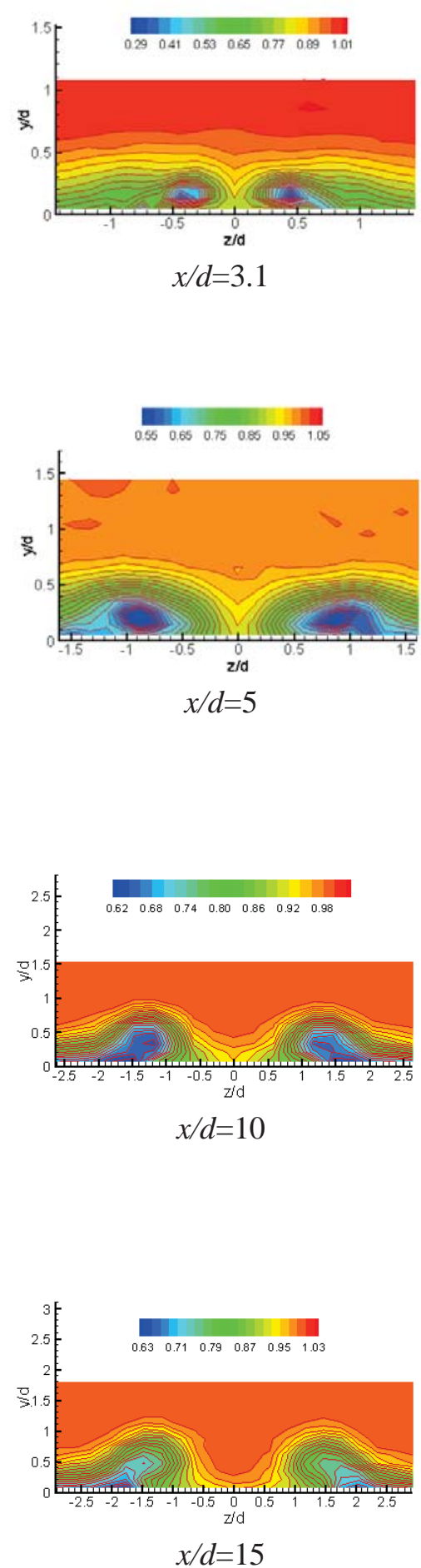

(b) VG only
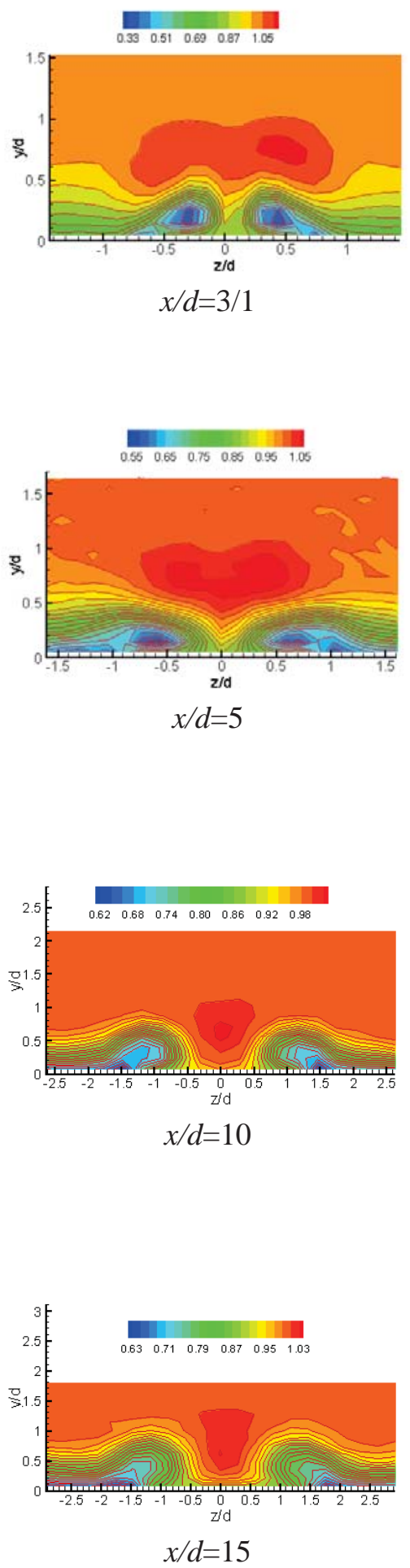

(c) VG plus JICF

Fig. 5 Mean velocity $\left(U / U_{\infty}\right)$ measured at indicated $x / d$ locations; VG 'H0(R0)' with tip at $x / d=1$. 

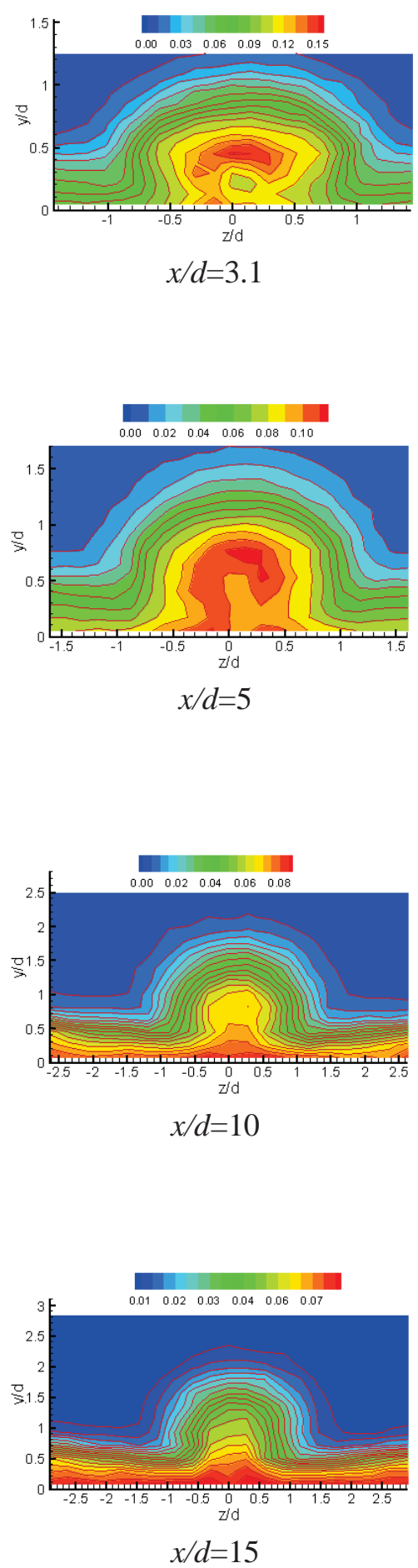

(a) JICF only
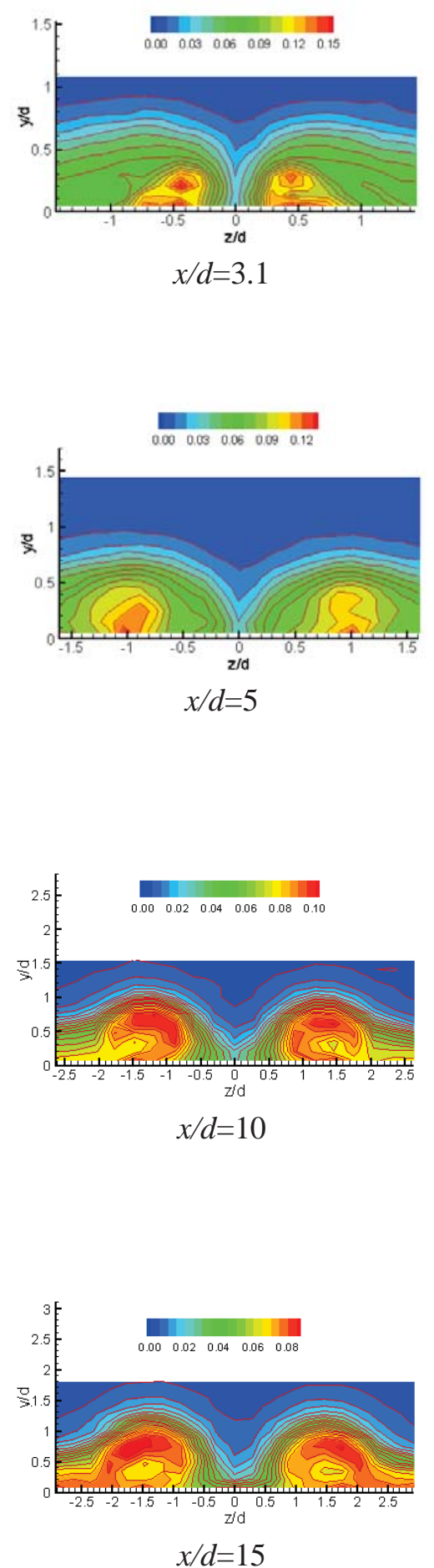

(b) VG only
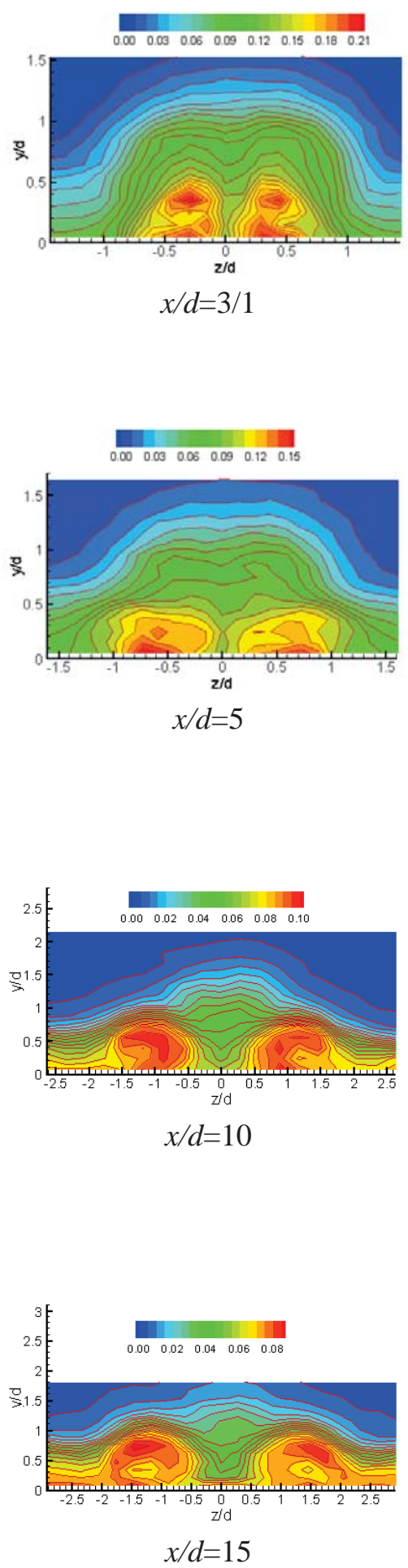

(c) VG plus JICF

Fig. 6 Turbulence intensity $\left(u^{\prime} / U_{\infty}\right)$ corresponding to the data of Fig. 5. 

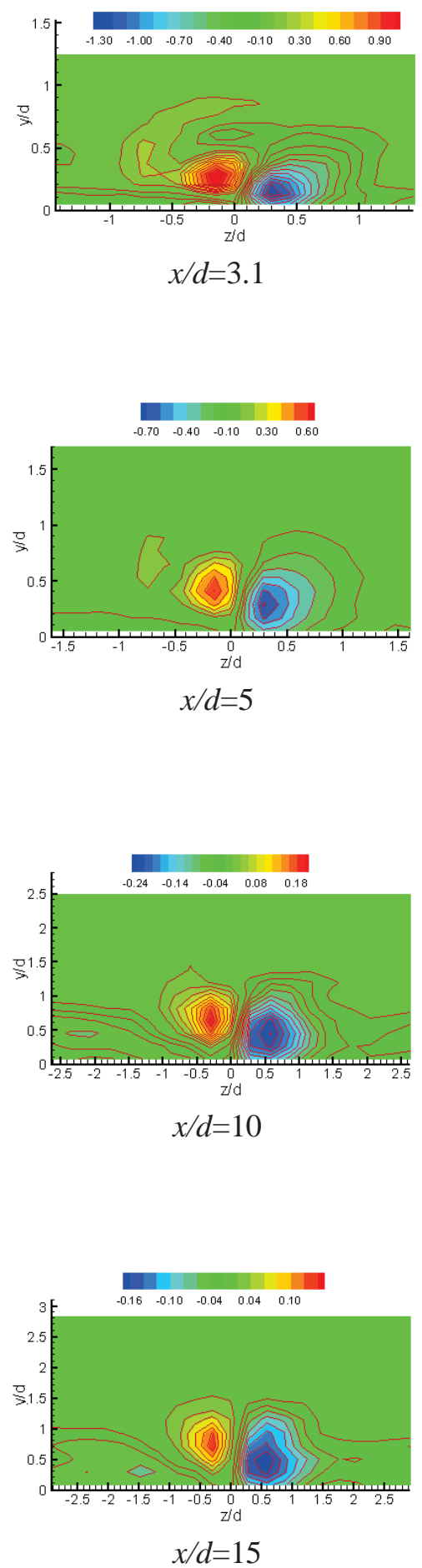

(a) JICF only
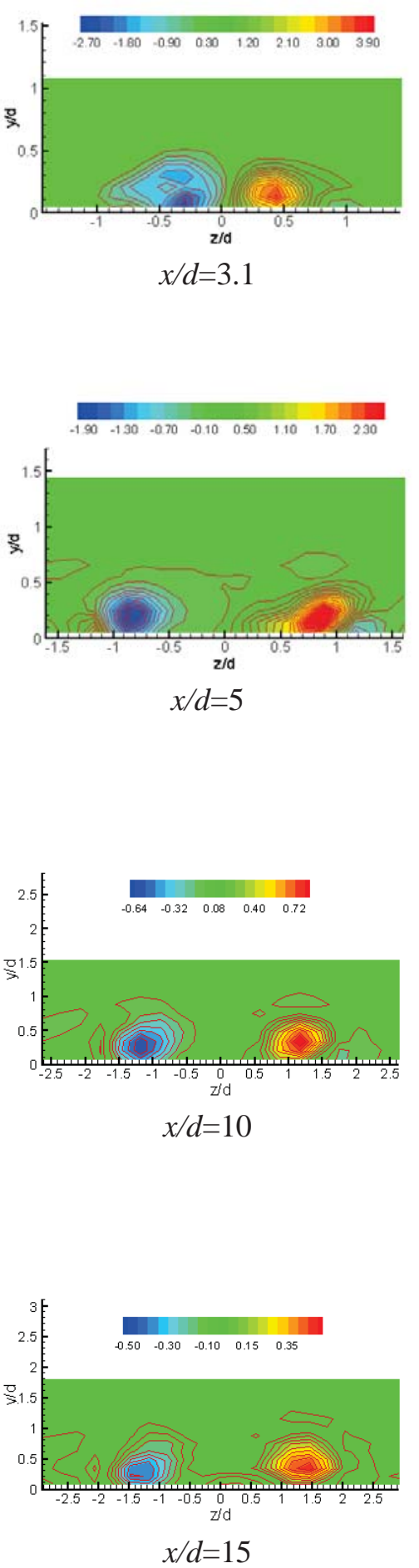

(b) VG only
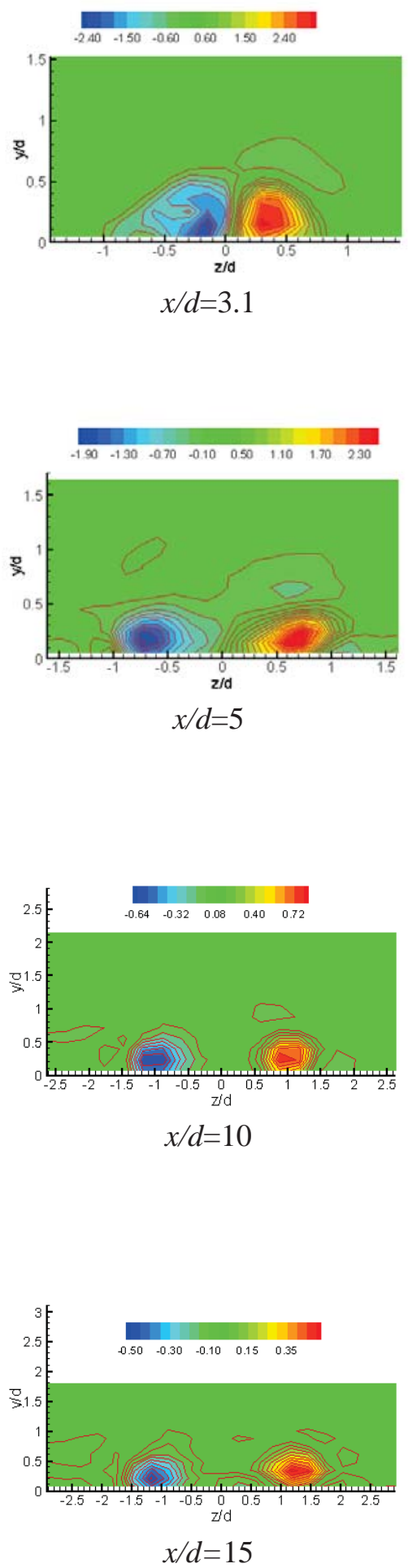

(c) VG plus JICF

Fig. 7 Streamwise vorticity $\left(\omega_{x} d / U_{\infty}\right)$ corresponding to the data of Fig. 5. 


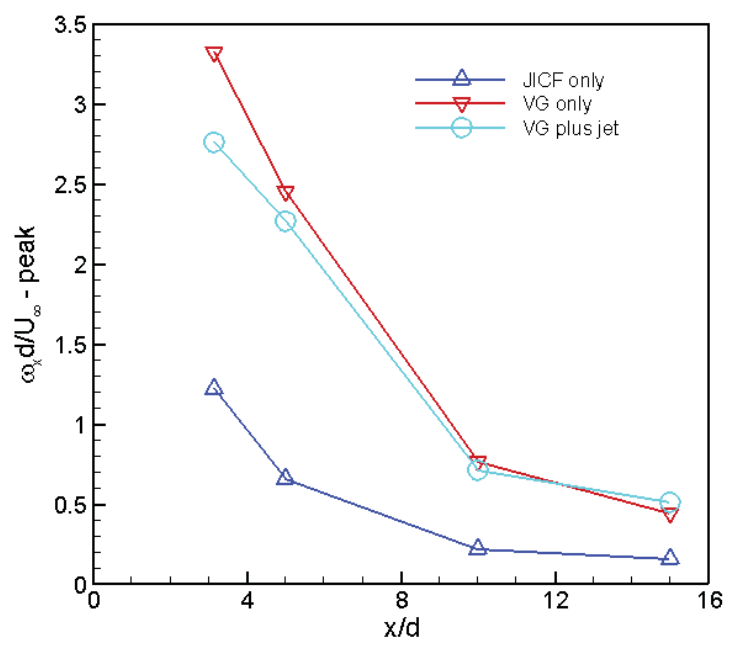

Fig. 8 Variation of peak streamwise vorticity with distance for indicated conditions.

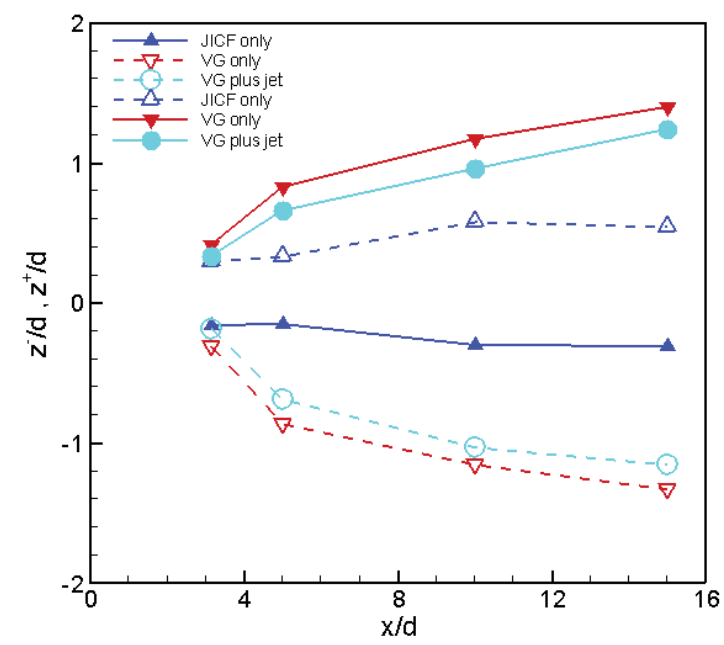

Fig. 9 Trajectories of streamwise vortex cores with distance for indicated conditions.

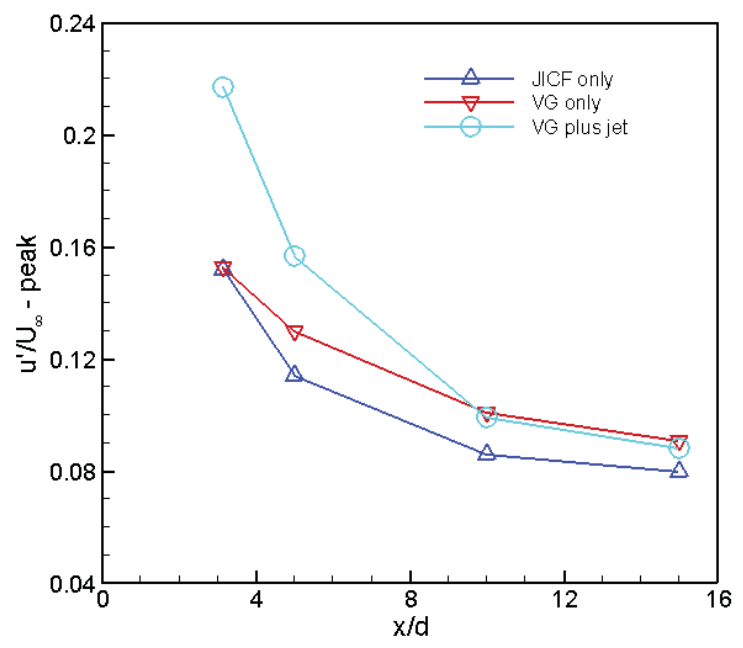

Fig. 10 Variation of peak turbulence intensity with distance for indicated conditions.

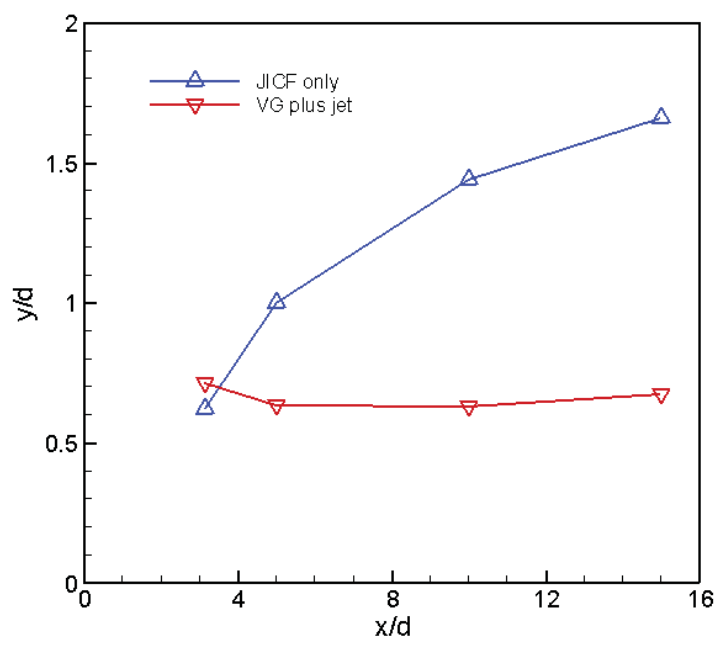

Fig. 11 Approximate trajectory of peak mean velocity ('jet penetration') for indicated conditions. 


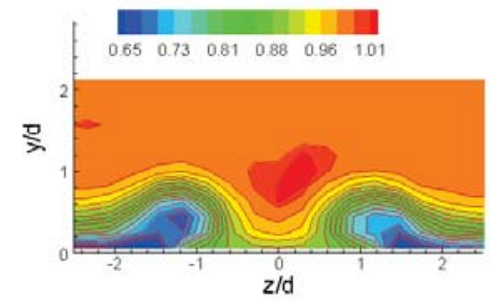

VG tip at $x / d=0$

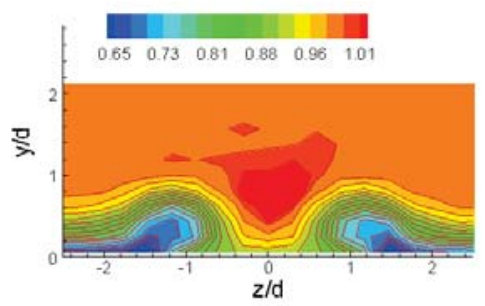

tip at $x / d=0.5$

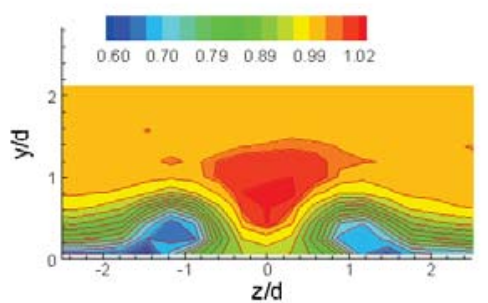

tip at $x / d=2$

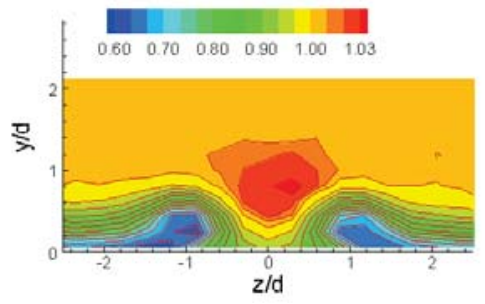

tip at $x / d=3$

(a) Velocity

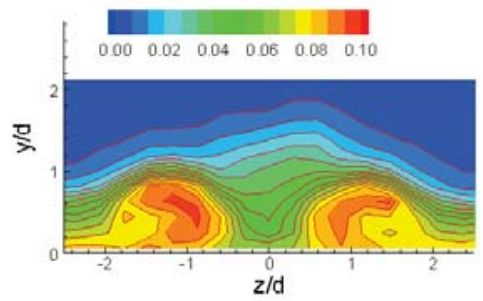

VG tip at $x / d=0$

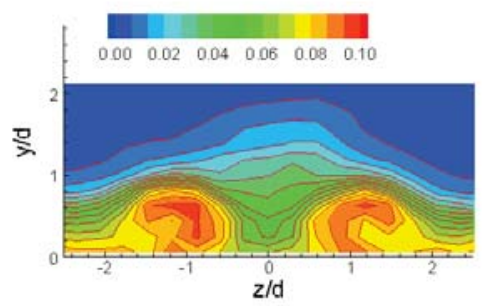

tip at $x / d=0.5$

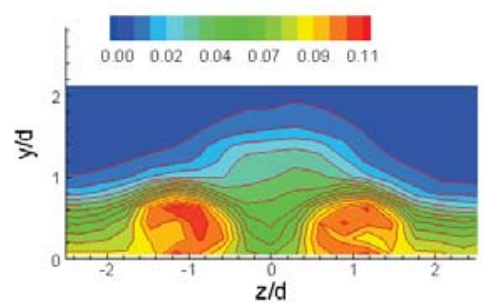

tip at $x / d=2$

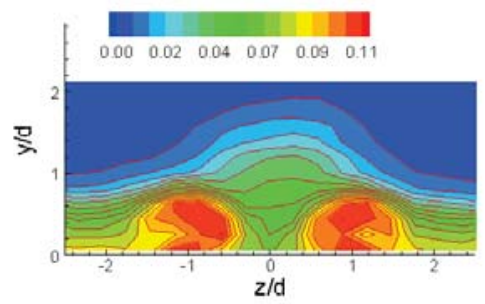

tip at $x / d=3$

(b) Turbulence intensity

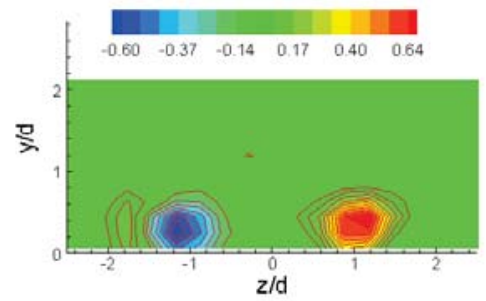

VG tip at $x / d=0$

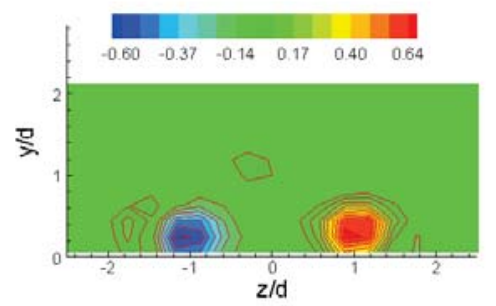

tip at $x / d=0.5$

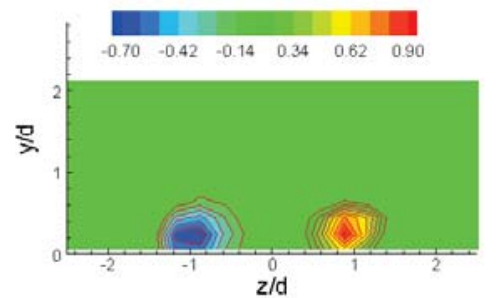

tip at $x / d=2$

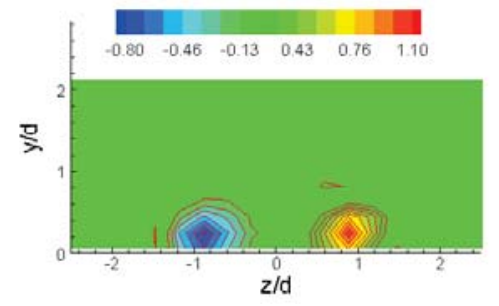

tip at $x / d=3$

(c) Streamwise vorticity

Fig. 12 Effect of VG location measured at $x / d=10$; (a) $U / U_{\infty}$, (b) $u^{\prime} / U_{\infty}$, (c) $\omega_{x} d / U_{\infty}$. 


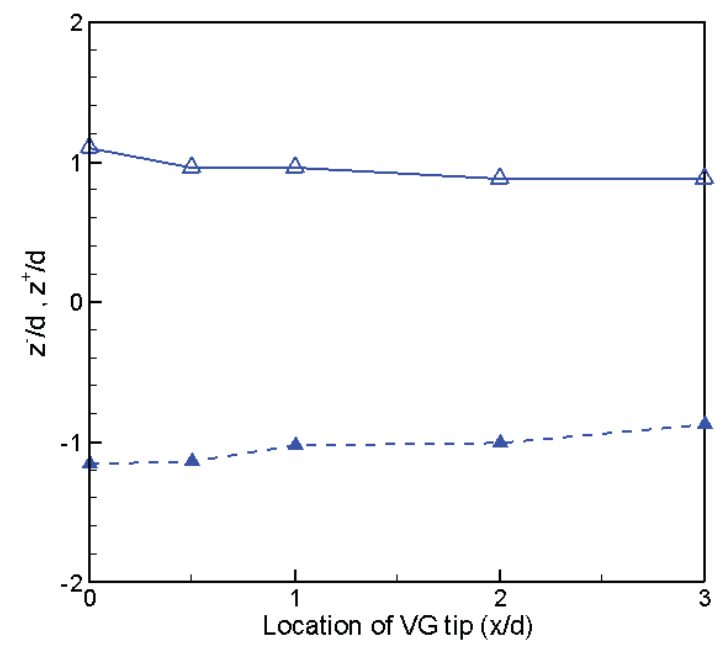

Fig. 13 Streamwise vortex core locations at $x / d=10$ with variation of VG location.

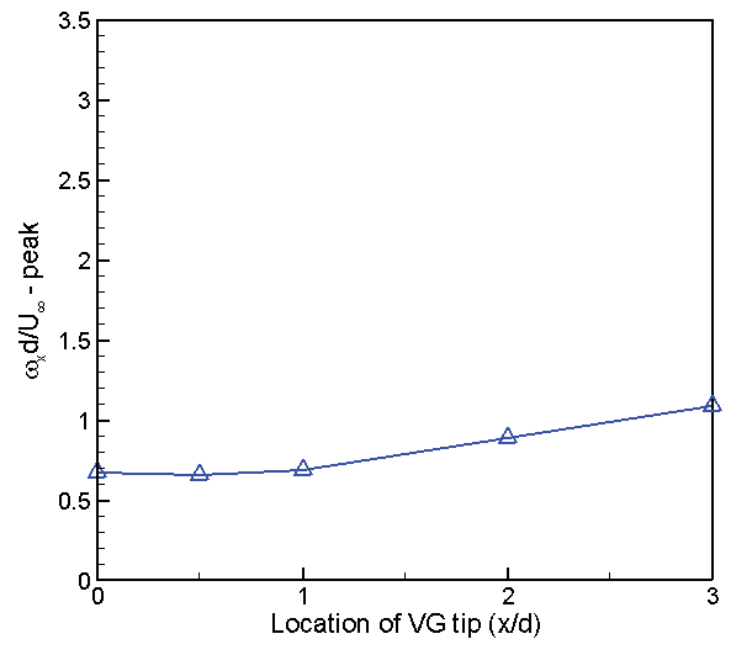

Fig. 14 Peak streamwise vorticity at $x / d=10$ with variation of VG location.
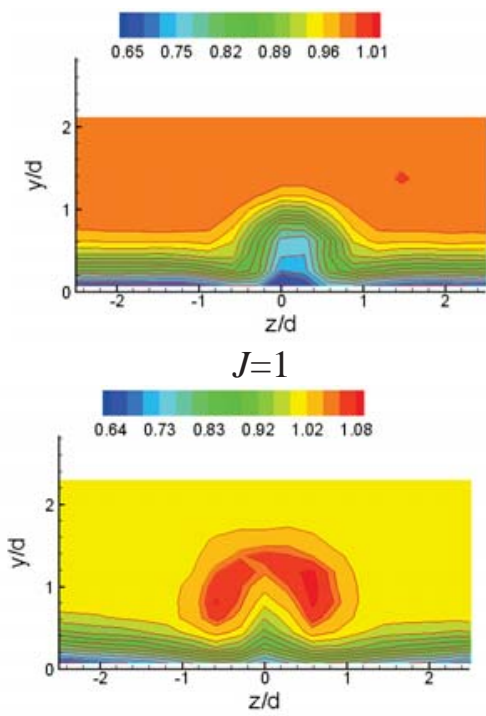

$J=3$

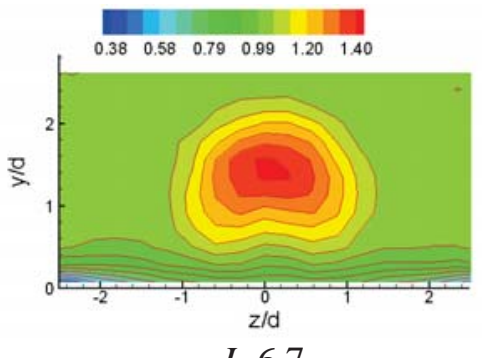

(a) Mean velocity

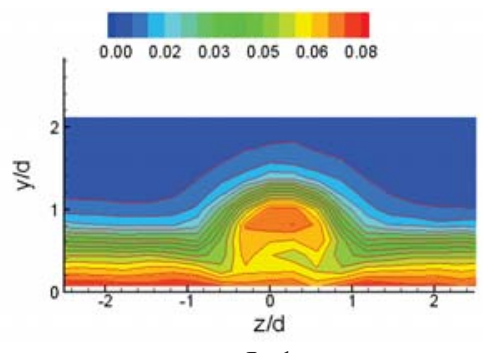

$J=1$

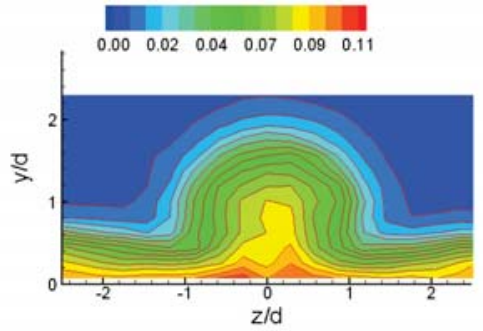

$J=3$

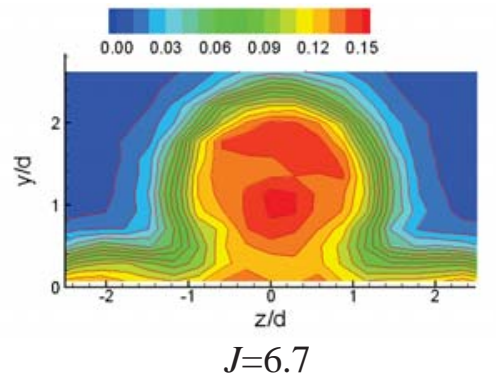

(b) Turbulence intensity
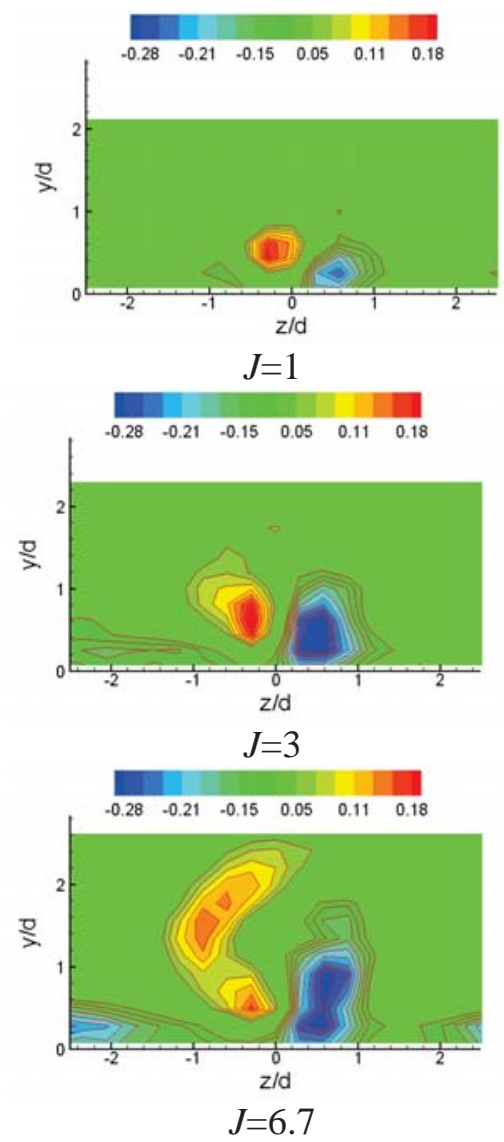

(c) Streamwise vorticity

Fig. 15 Effect of VG at different momentum flux ratio ( $J$ ). Measurement at $x / d=10$ for JICF-only; (a) $U / U_{\infty}$, (b) $u^{\prime} / U_{\infty}$, (c) $\omega_{x} d / U_{\infty}$. 

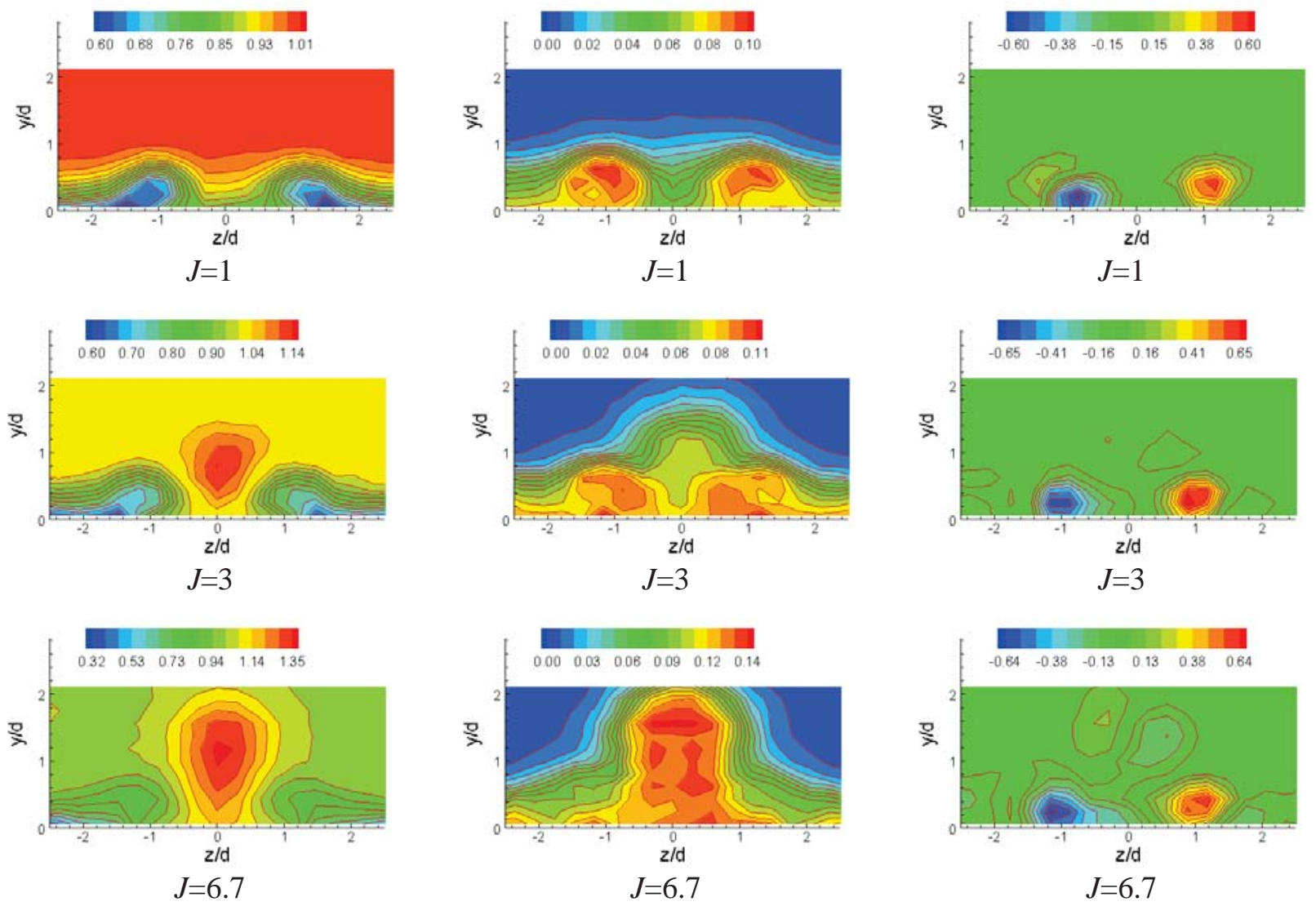

(a) Mean velocity

(b) Turbulence intensity

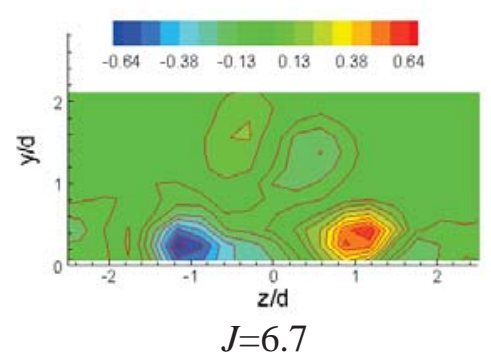

Fig. 16 Effect at different momentum flux ratio $(J)$. Measurement at $x / d=10$ for VG plus JICF; (a) $U / U_{\infty}$, (b) $u^{\prime} / U_{\infty}$, (c) $\omega_{x} d / U_{\infty}$.

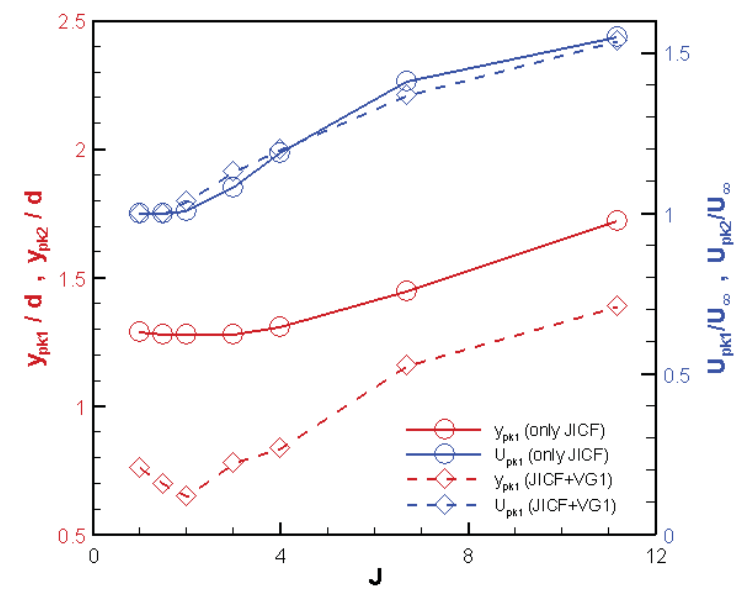

Fig. 17 Effect of $J$ on location and magnitude of $U$-peak with and without VG; ordinate for magnitude on right. 


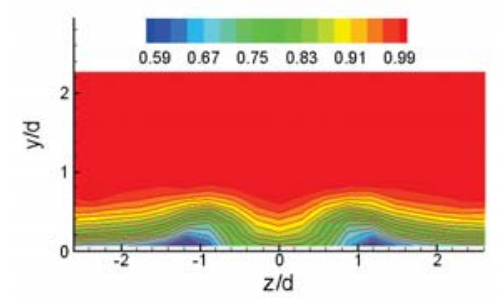

$H / d=0.375$

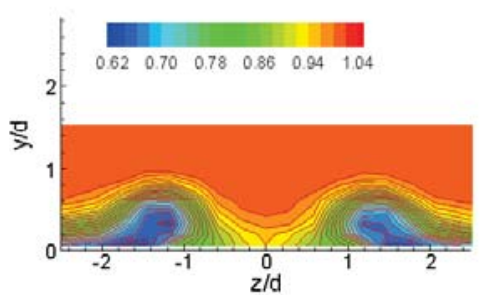

$H / d=0.75$

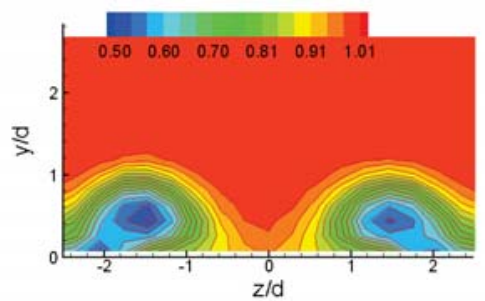

$H / d=1.50$

(a) Mean velocity

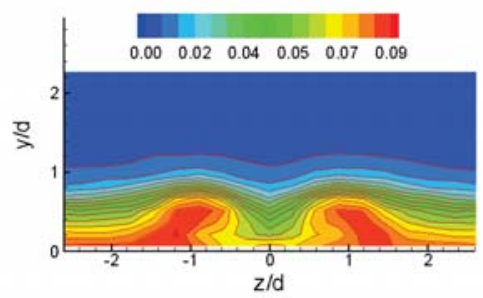

$H / d=0.375$

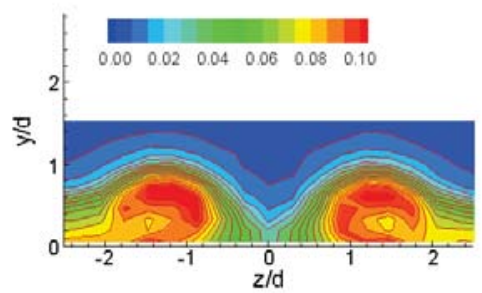

$H / d=0.75$

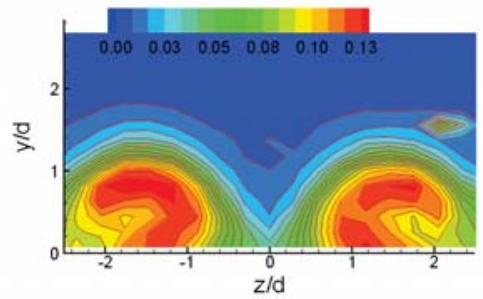

$H / d=1.50$

(b) Turbulence intensity

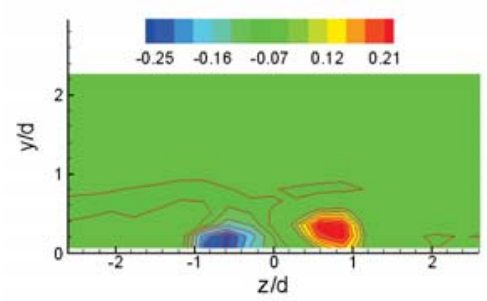

$H / d=0.375$

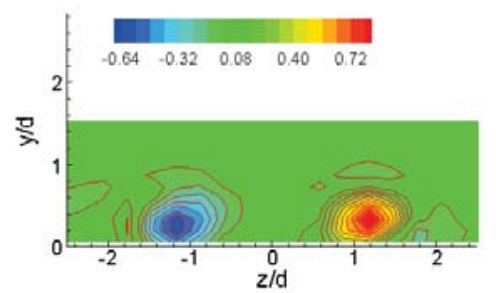

$H / d=0.75$

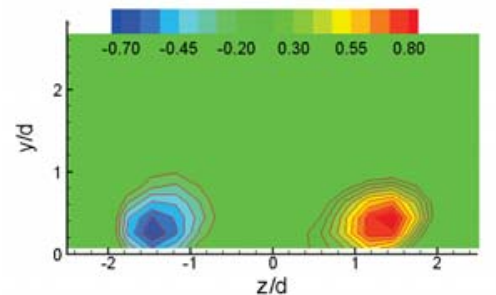

$H / d=1.50$

(c) Streamwise vorticity

Fig. 18 Effect of VG height $(H)$. Measurement at $x / d=10$ for VG only; (a) $U / U_{\infty}$, (b) $u^{\prime} / U_{\infty}$, (c) $\omega_{x} d / U_{\infty}$. 


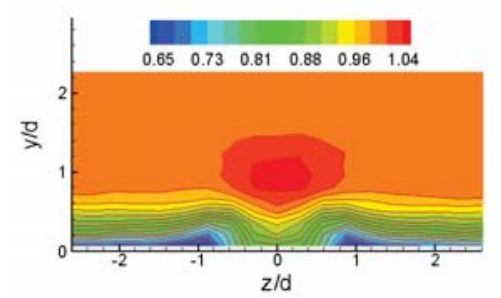

$H / d=0.375$

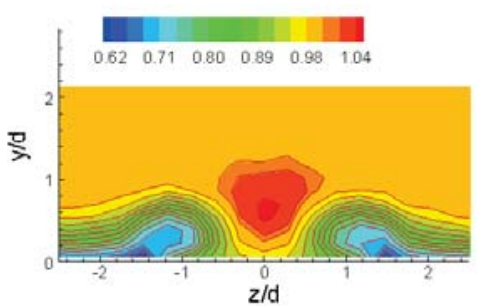

$H / d=0.75$

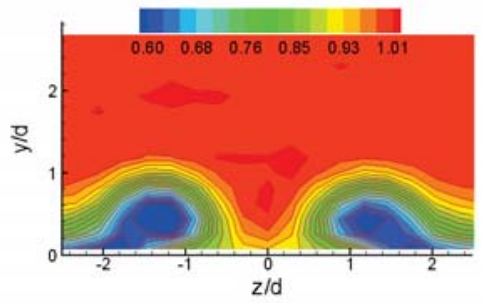

$H / d=1.50$

(a) Mean velocity

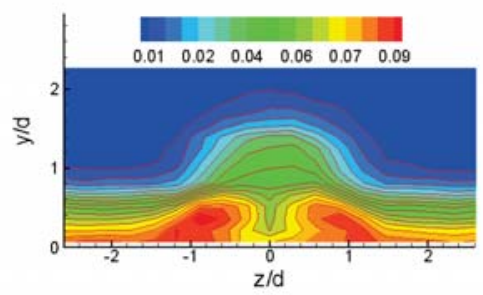

$H / d=0.375$

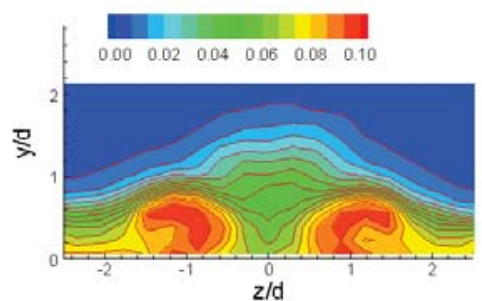

$H / d=0.75$

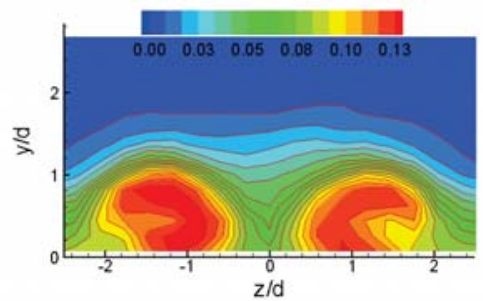

$H / d=1.50$

(b) Turbulence intensity

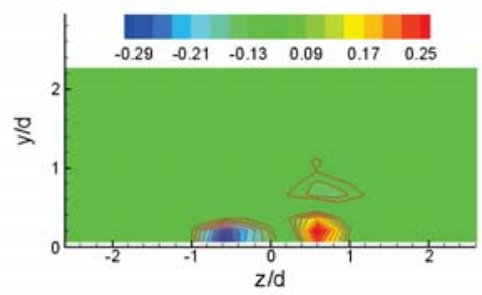

$H / d=0.375$

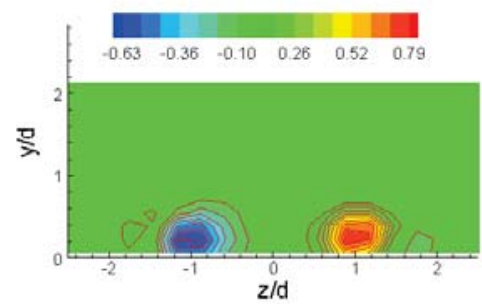

$H / d=0.75$

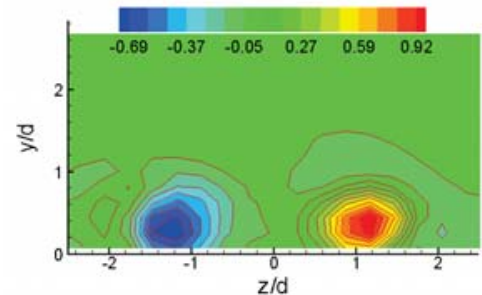

$H / d=1.50$

(c) Streamwise vorticity

Fig. 19 Effect of VG height $(H)$. Measurement at $x / d=10$ for VG plus JICF; (a) $U / U_{\infty}$, (b) $u^{\prime} / U_{\infty}$, (c) $\omega_{x} d / U_{\infty}$ 

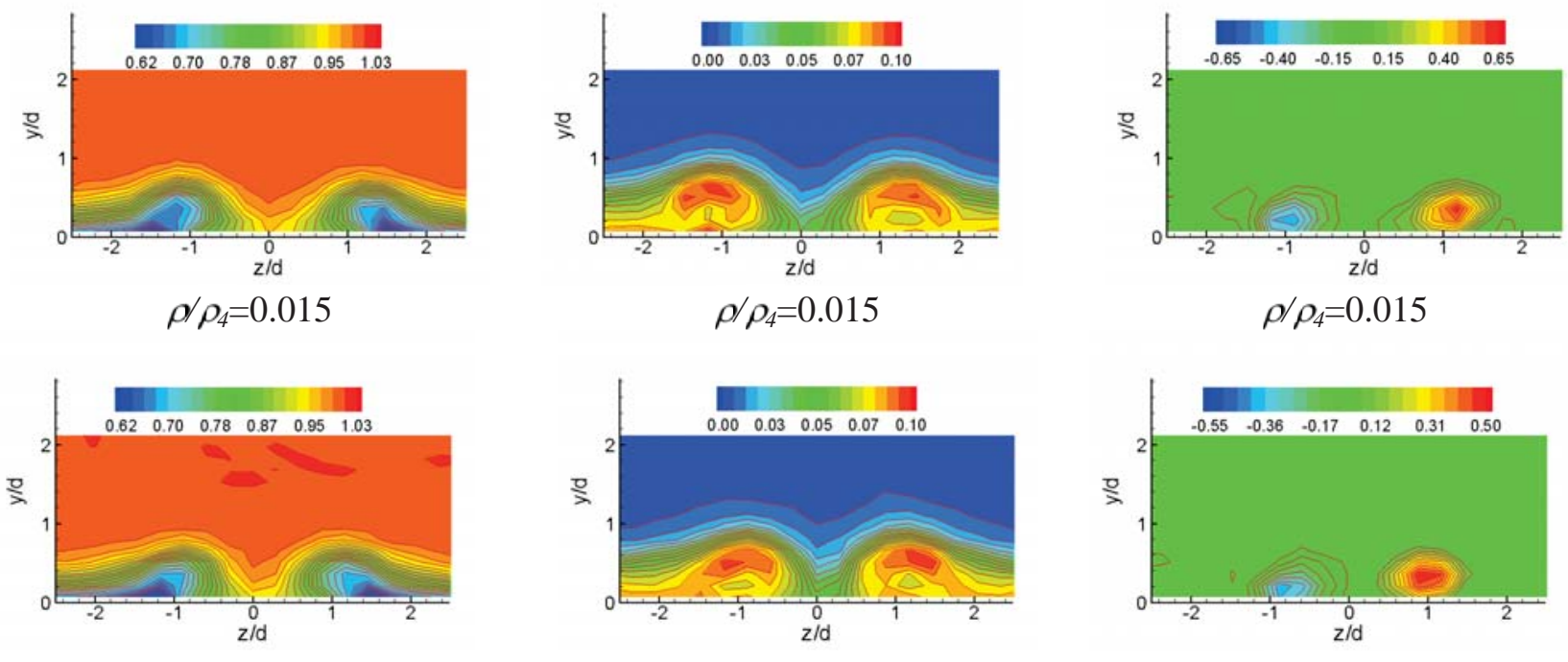

$\rho / \rho_{4}=0.415$

$\rho / \rho_{4}=0.415$

$\rho / \rho_{4}=0.415$

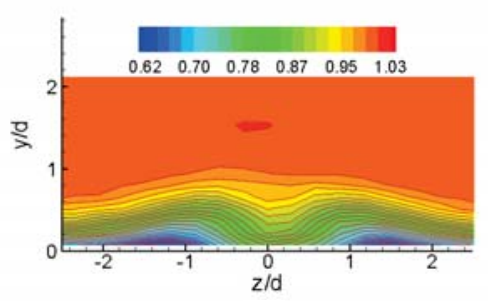

$\rho / \rho_{4}=1$

(a) Mean velocity

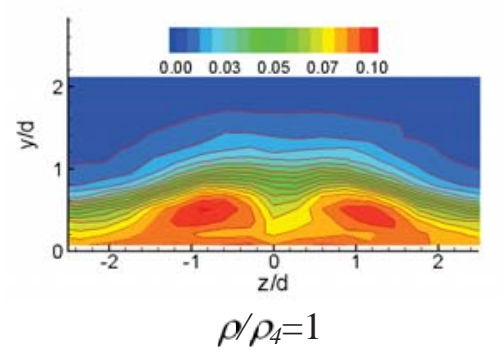

(b) Turbulence intensity

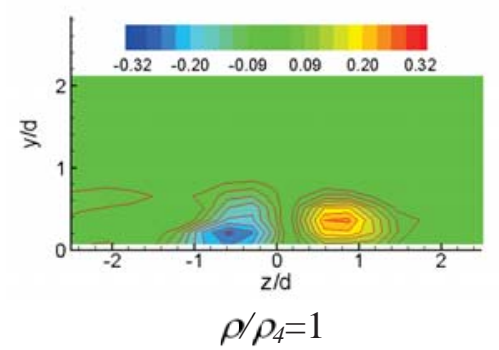

(c) Streamwise vorticity

Fig. 20 Effect of rounding off the edges of the VG $\left(\rho / \rho_{4} ; \rho_{4}\right.$ is radius of curvature for the fully rounded model R4). Measurement at $x / d=10$ for VG only; (a) $U / U_{\infty}$, (b) $u^{\prime} / U_{\infty}$, (c) $\omega_{x} d / U_{\infty}$. 

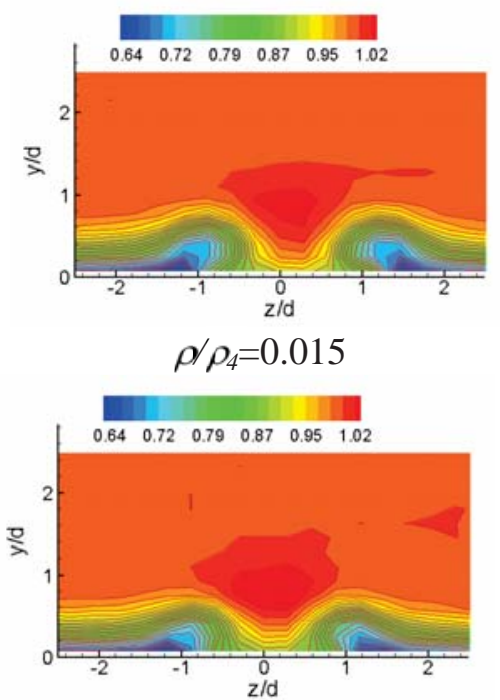

$\rho / \rho_{4}=0.415$

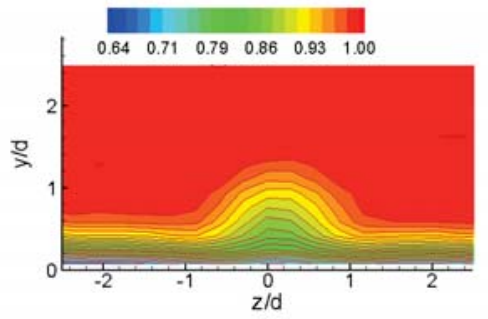

$\rho / \rho_{4}=1$

(a) Mean velocity

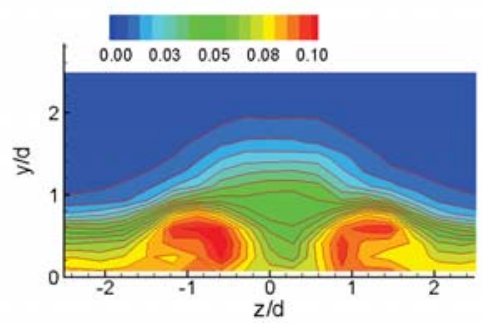

$\rho / \rho_{4}=0.015$

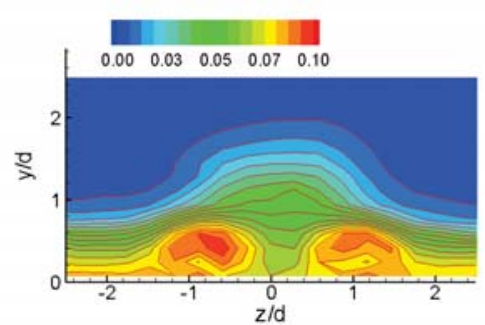

$\rho / \rho_{4}=0.415$

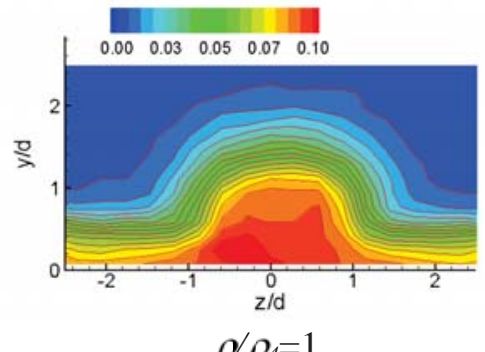

(b) Turbulence intensity

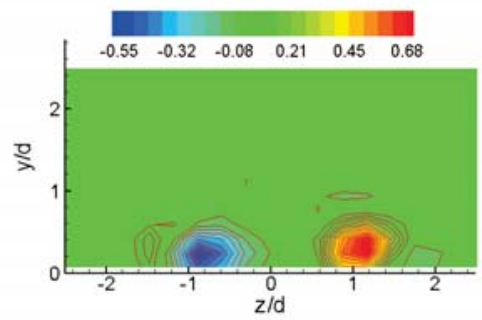

$\rho / \rho_{4}=0.015$

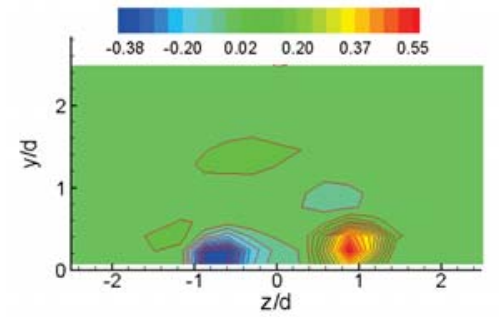

$\rho / \rho_{4}=0.415$

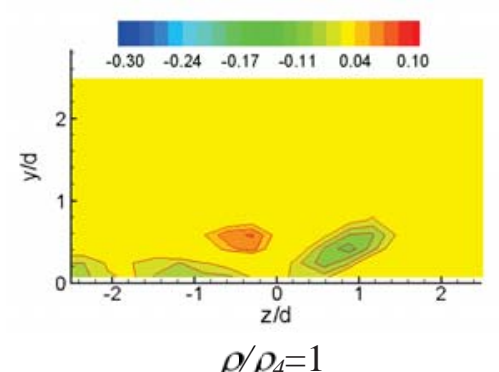

(c) Streamwise vorticity

Fig. 21 Effect of rounding off the edges of VG; VG plus JICF, $x / d=10$. (a) $U / U_{\infty}$, (b) $u^{\prime} / U_{\infty}$, (c) $\omega_{x} d / U_{\infty}$

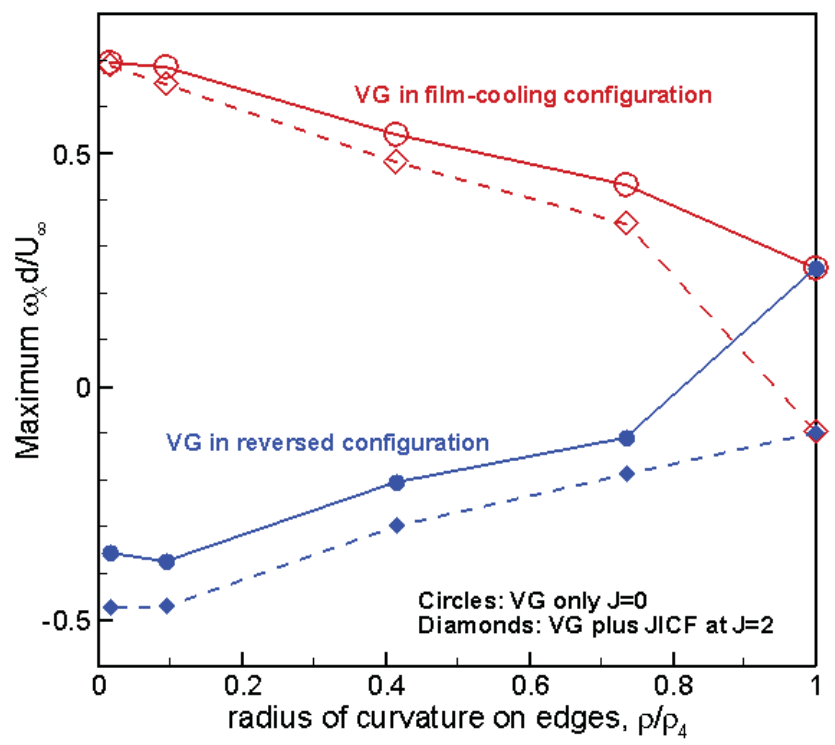

Fig. 22 Effect of rounding off the edges of VG on peak streamwise vorticity at $x / d=10$. Red curves are for 'film-cooling' configuration of VG as used for all previous data. Blue curves are for configuration where VG orientation is reversed by $180^{\circ}$. Solid curves: VG-only, dashed curves: VG-plus-JICF. 